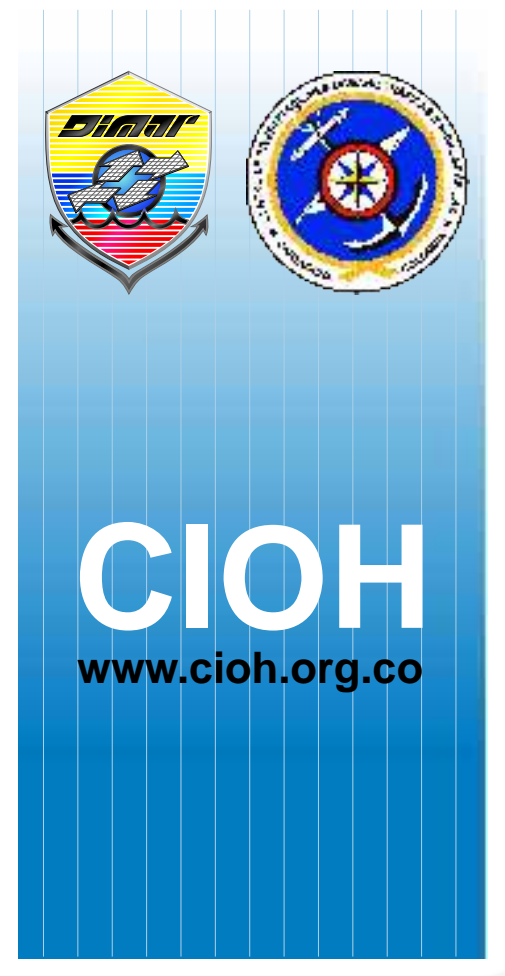

ARTíCULO

\title{
Caracterización del patrón de circulación de las bahías Sapzurro y Capurganá mediante la implementación de un modelo hidrodinámico en dos dimensiones
}

\author{
Circulation pattern characterization of Sapzurro and \\ Capurganá bays by implementing a two-dimensional \\ hydrodynamic model
}

Fecha recepción: 2008-07-28 / Fecha aceptación: 2008-09-02

\section{Resumen}

Isabel Cristina Hoyos Rincón, ihoyos@ fisica.udea.edu.co Tomás Ramón Florville Alejandre, inuca@quimbaya.udea.com Carlos Alberto Palacio Tobón, cpalacio@udea.edu.co Universidad de Antioquia, Medellín

En este estudio, se presentan los resultados de la aplicación del modelo hidrodinámico en dos dimensiones, RMA, en régimen turbulento a las bahías Sapzurro y Capurganá en el Urabá chocoano. Se simulan diversos escenarios y períodos donde se incluyen los efectos de viento y marea. La sensibilidad de los parámetros físicos y numéricos es determinada por el método de perturbación de parámetros. La calibración y validación del modelo es soportada por observaciones obtenidas del monitoreo de boyas de deriva durante dos fases de campo correspondientes a épocas distintas. El modelo permite caracterizar el patrón general de circulación para la zona e identificar áreas potenciales de erosión y transporte de sedimentos.

Palabras claves: Oceanografía costera, circulación del agua, propiedades del agua, modelos matemáticos, condiciones de frontera, boyas de deriva, erosión costera.

\section{Abstract}

This study presents the results of two-dimensional RMA hydrodynamic model in turbulent regime implemented on the bays Sapzurro and Capurganá in Urabá chocoano. Various scenarios and time periods are simulated, which include the effects of tide and wind. The sensibility of the numerical and physical parameters is determined by the method of disturbance parameters. The calibration and validation of the model are supported by field observations obtained from monitoring of drifting buoys during two different field phases. The model allows characterize the general water motion pattern for the zone and identify potential areas of erosion and sediment transport.

Key words: Coastal oceanography, water circulation, water properties, mathematical models, boundary conditions, drifting buoys, coastal erosion. 


\section{Introducción}

El aumento global en la población es considerado actualmente como un factor significativo en la conservación, la calidad y la explotación de los recursos marinos y costeros [1]. En Colombia, el Comité Nacional de Manejo Integrado de Zonas Costeras, coordinado por la Comisión Colombiana del Océano [2], se ha encargado de promover normas y estrategias que vinculen y comprometan a los municipios de las zonas costeras del Pacífico, el Caribe y la zona insular del país, en el ordenamiento de los ecosistemas marinos y costeros y los factores bióticos y abióticos importantes para el hombre. De modo que, el estudio de las regiones costeras proporciona un amplio campo de investigación en diversas áreas de las ciencias y por lo tanto es oportuna la realización de estudios interdisciplinarios que aumenten el conocimiento sobre el estado, comportamiento y evolución de los procesos físicos que tienen lugar en estas localidades. La modelación es una herramienta fundamental para la caracterización de los patrones de circulación en diversos escenarios climáticos [3,4]; estos modelos se utilizan como base para simulación de transporte de contaminantes, difusión de derrame de hidrocarburos, monitoreo de objetos perdidos o a la deriva, construcción de obras civiles, determinación de conectividad genética entre peces de arrecife, procesos erosivos en el litoral, entre otros.

Los trabajos de investigación en el campo de la modelación hidrodinámica en las regiones costeras del país son escasos. Recientemente para el Caribe colombiano, en el marco del proyecto "Modelación Numérica Aplicada a Operaciones Navales de Búsqueda y Rescate", del CIOH (Centro de Investigaciones Oceanográficas e Hidrográficas), se construyó un sistema llamado SPOD (Sistema Predicción Oceánica de la Dirección General Marítima - DIMAR) que, acoplado al modelo existente POM (Princeton Ocean Model), [5,6] fue capaz de predecir condiciones oceanográficas hasta por 72 horas para el mar Caribe, con mayor resolución para las aguas de jurisdicción colombiana. Este modelo cuenta con una interfaz gráfica amigable, y es empleado para simular desplazamiento de objetos flotantes y movimiento de manchas de hidrocarburos, por lo que se perfila como una herramienta útil en operaciones de búsqueda y rescate, así como en planes de contingencia para derrames de hidrocarburos, entre otros.

En el golfo de Urabá, Molina, et al. [7] proponen una descripción de las corrientes superficiales para las dos épocas climáticas dominantes -secas (diciembreabril) y lluvias (mayo-noviembre). Con base en imágenes de satélite y la observación del transporte de sedimentos en la pluma del río Atrato, sugieren una corriente que ingresa al golfo por el extremo occidental en dirección N-S y una corriente que sale del mismo por el extremo oriente en dirección S-N, determinando el sentido de la corriente de deriva litoral en su interior. En [8] se presenta un estudio hidrológico del golfo de Urabá, empleando la misma técnica de análisis de imágenes satelitales utilizada en [7] y adicionalmente se estudian variaciones de salinidad para las temporadas de lluvias y secas, encontrándose que las mayores salinidades se registran en época de lluvias. Este comportamiento atípico se explica como consecuencia del patrón de circulación de vientos que caracteriza a la zona (durante temporada seca, fuertes vientos del norte y noreste confinan el agua de los ríos en el interior del golfo). En la Escuela de Geociencias y Medio Ambiente de la Universidad Nacional de Colombia, $[9,10]$ implementan un modelo hidrodinámico para el golfo de Urabá con datos obtenidos de la campaña oceanográfica Urabá I. Esta investigación reporta una fuerte estratificación salina generada por el Atrato, que induce circulación superficial saliente del golfo hacia el extremo noroccidental. Adicionalmente, en [9] se muestran las principales componentes de marea para la zona, con datos obtenidos de la estación meteorológica "San Cristóbal", Panamá y en [10] se relaciona la corriente de deriva litoral con el transporte de sedimentos para las costas entre Cabo Tiburón y Acandí.

El trabajo presentado, pretende hacer un aporte al conocimiento del estado de las costas colombianas, mediante la aplicación de un modelo hidrodinámico a las bahías Sapzurro y Capurganá en el Urabá chocoano que tal como se observa en [11] exhibe una franja litoral rica en biodiversidad y un creciente problema de erosión costera. Se presentan aquí las hipótesis físicas para el estudio preliminar de esa región, y los pasos para la implementación a este caso específico del modelo genérico RMA (modelo numérico utilizado para la simulación hidrodiná- 
mica). Es importante destacar que este es el primer trabajo realizado en Colombia donde la información generada del modelo es confrontada con datos de campo obtenidos por monitoreo de boyas de deriva y no existen referentes anteriores al presente trabajo sobre la circulación de las aguas en el interior de estas bahías. Finalmente la implementación de este modelo, permite caracterizar el patrón de circulación para la región en diferentes condiciones oceanográficas y climatológicas estableciendo la importancia relativa entre los factores que influyen en el movimiento del agua y generando mapas de distribución espacial para el campo de velocidad en diferentes fases de marea.

\section{Área de estudio}

Las bahías de Capurganá y Sapzurro están ubicadas en el noroccidente del golfo de Urabá, en el departamento del Chocó, en la frontera Panamá-Colombia y bajo la jurisdicción del municipio de Acandí, figura 1. Con una distribución espacial entre $1445000 \mathrm{~N}$ $1451000 \mathrm{~N}$ y $967600 \mathrm{E}$ 972500E en coordenadas planas (en coordenadas geográficas el área de estudio se extiende desde $8^{\circ} 40^{\prime} 30^{\prime \prime} \mathrm{N}, 7^{\circ} 21^{\prime} 45^{\prime \prime} \mathrm{E}$ hasta $8^{\circ} 37^{\prime} 20^{\prime \prime} \mathrm{N}, 77^{\circ} 19^{\prime} 30 " \mathrm{E}$ ), correspondientes respectivamente a Cabo Tiburón y Punta del Aguacate. Esta región cubre una extensión de aproximadamente 10 $\mathrm{km}$ de línea de costa. Por su ubicación en el Caribe colombiano, se encuentra en la zona de convergencia intertropical, donde confluyen corrientes subtropicales de ambos hemisferios. Cuando esta "franja" se sitúa sobre Centro y Suramérica (mayonoviembre), se presentan fuertes lluvias y vientos débiles en el Caribe colombiano con direcciones variables pero con predominio de las componentes del sur y bajas velocidades, cuando se sitúa en el hemisferio sur (diciembre - abril), se presentan fuertes vientos en dirección $\mathrm{N}$ y $\mathrm{NE}$ llamados los vientos Alisios con promedios mensuales de $4 \mathrm{~m} / \mathrm{s}$, siendo más intensos en febrero, cuando el promedio alcanza los $9.4 \mathrm{~m} / \mathrm{s}$ [8] y épocas de baja lluviosidad [12].

El oleaje está fuertemente influenciado por el viento, por lo cual depende de la época climática, presentándose mayor perturbación en época seca, con altura de olas máxima de hasta $2.5 \mathrm{~m}$ y en promedio $0.5 \mathrm{~m}$ de acuerdo a la clasificación de Beaufort y su equivalente en la escala de Douglas presentadas en [13].
Esta región, corresponde en la clasificación de zonas de vida de Holdridge, a Bosque Húmedo Tropical, descrita como una de las más lluviosas del mundo [8].

Según IDEAM [12] y Velásquez [11], presenta un régimen de lluvias bimodal con dos temporadas muy lluviosas (mediados de abril hasta agosto y octubre hasta noviembre) con dos períodos un poco menos lluviosos (diciembre a mediados de abril y septiembre).

El régimen de marea para el golfo de Urabá, según [9] es micromareal con amplitudes inferiores a $40 \mathrm{~cm}$. Del análisis de las series de tiempo de elevación para la zona, tomadas del programa computacional de libre distribución WXTide el comportamiento anual se clasifica como mixto y semidiurno.

Respecto a las características termodinámicas, se puede determinar de los datos de temperatura y salinidad registrados en Correa [14] durante septiembre de 2004 y septiembre de 2005, una salinidad promedio de $35.5 \%$ y una temperatura superficial promedio de $29.9^{\circ} \mathrm{C}$. De acuerdo con estos datos, la densidad estimada de la ecuación de estado del agua de mar determinada por la UNESCO en GOFUVI[13] es $1022.138 \mathrm{~kg} / \mathrm{m}^{3}$.

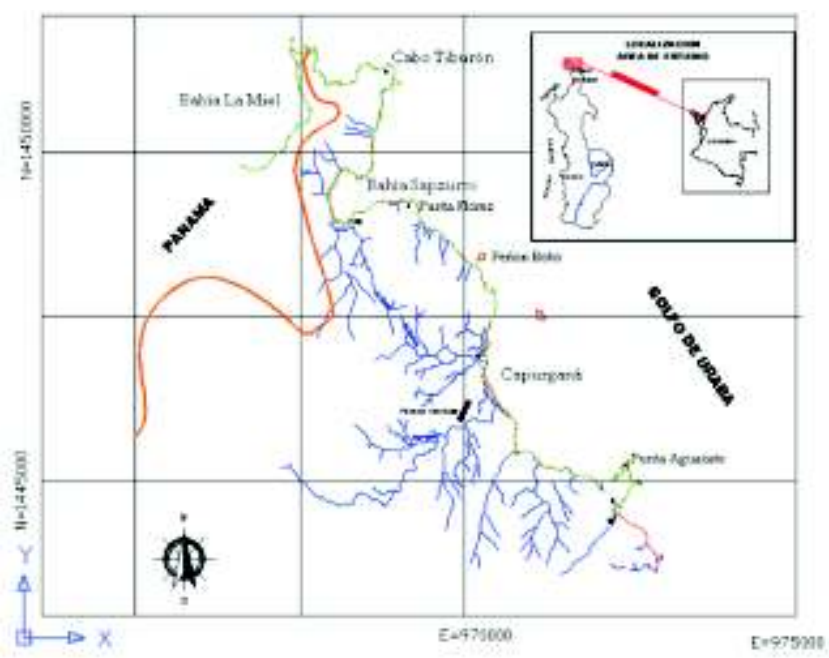

Figura 1. Ubicación geográfica del área de estudio. Modificado de Velásquez [11].

En esta región se presenta predominio de aguas oceánicas reflejado en las condiciones termodiná- 
micas y la formación de esponjas y arrecifes de coral $[9,11,15]$. A gran escala, está influenciada principalmente por el giro ciclónico Panamá-Colombia denominado contracorriente del Darién en la zona costera, que a pesar de tener un flujo y dirección variable estacionalmente, en el área de interés permanece en dirección E todo el año [16,17].

Morfológicamente, Sapzurro es una bahía muy cerrada y profunda, que se distingue por tener extensiones muy amplias de plataformas rocosas de poca profundidad, interrumpidas por caños y caídas rocosas entre los 10 y $30 \mathrm{~m}$ de profundidad, con un variado paisaje submarino, formaciones arrecífales y zonas de thalasia. La zona de Cabo Tiburón, presenta acantilados con rocas de origen volcánico con un rápido incremento en la profundidad, alcanzando profundidades de $60 \mathrm{~m}$ cerca del cabo, mezclados con playas de arenas blancas. La bahía de Capurganá presenta un panorama geomorfológico similar al de Sapzurro con menores profundidades y más abierta [11].

La dinámica litoral del sector está afectada enormemente por la morfología del lugar, la desviación de la dirección del frente de olas al acercarse a las playas y las zonas de rompientes están influenciadas por la formación de barreras y bajos coralinos, produciéndose una corriente de deriva litoral generada por el oleaje y controlada por el viento, que circula paralela a la línea de costa y participa activamente en su evolución [18].

\section{Metodología}

\section{Recopilación y digitalización de la información de contornos geográficos y condiciones de frontera}

Mediante una tableta digitalizadora adaptada al programa computacional AUTOCAD, se digitalizó la carta náutica Col 280, que cubre la región Cabo Tiburón a Isla Terrón de Azúcar, para la cual, los levantamientos hidrográficos fueron efectuados por el Centro de Investigaciones Oceanográficas e Hidrográficas DIMAR - ARC, con la línea de costa basada en la carta Col 412, 1ra ed. dic 1997 y con escala 1:25.000. Con el objetivo de refinar la línea de costa por identificación de zonas de bajamar, esta información se integra mediante un Sistema de Información Geográfica (SIG) desarrollado con el programa computacional ArcGIS, a la imagen satelital
042-454 tipo LandSat, distribuída gratuitamente en internet por Global Land Cover Facility Herat Science Data Interface. Con los datos de la carta náutica y otros tomados en campo, se reduce la batimetría a un tamaño de celda de 25 m mediante el método IDW (Inverse Distance Weigth), con potencia 3 y con 12 vecinos [19], como se presenta en la figura 2.

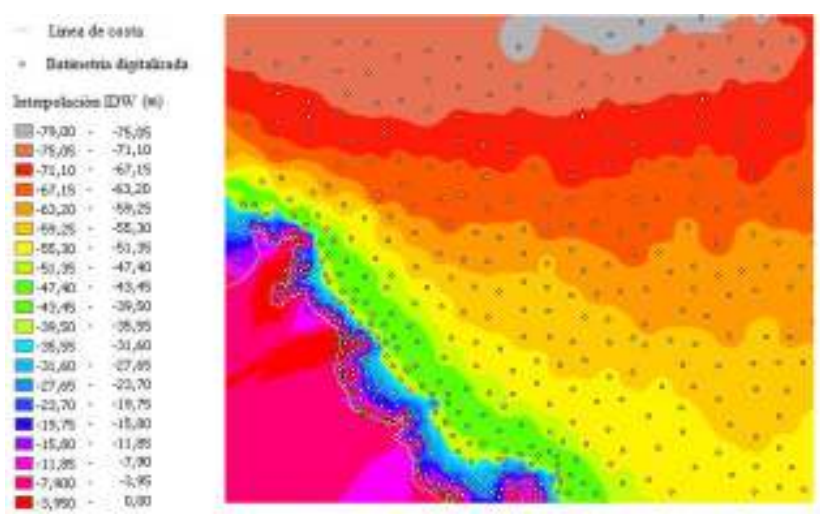

Figura 2. Interpolación batimétrica del área de estudio con el método Inverse Distance Weigth (IDW). Los valores de profundidad se dan en metros. La resolución de la batimetría a partir de la cual se realiza la interpolación se ilustra con círculos.

Los niveles de elevación del agua se obtienen de series de tiempo generadas por el programa computacional de pronóstico de mareas WXTide32 de distribución gratuita, además de los armónicos de marea más importantes para la zona, presentados en [9].

\section{Información de campo}

Para obtener un perfil de velocidades reales para la zona se construyeron 6 boyas de deriva en forma de crucetas como sugieren Vachon [20] y Davis [21] para ser monitoreadas y registrar la variación de su posición en el tiempo durante varios ciclos de marea, figura 3. La elección de estos dispositivos obedece principalmente a tres razones: bajo costo, se pueden vigilar fácilmente desde una pequeña embarcación y permiten la visualización real de patrones de flujo horizontal.

El proceso consiste en elegir puntos estratégicos dentro y alrededor de las bahías para iniciar el monitoreo, una vez se ha llegado al sitio, se abandona la estructura registrando la posición y la hora de un GPS (Global Position System), luego se registra 
sucesivamente la evolución espacial y temporal de cada cuerpo de deriva. Esta información es integrada al SIG. La velocidad media sobre un período es calculada, como la razón de la distancia entre el punto inicial y final y el tiempo que tarda para recorrer dicha distancia [4]. Este procedimiento se realizó para las dos bahías desde Cabo tiburón hasta la Punta del Aguacate, en dos fases de campo; la primera del 10 al 13 de octubre de 2006 con un total de 11 lanzamientos y la segunda del 18 al 22 de diciembre de 2006 con un total de 33 lanzamientos.
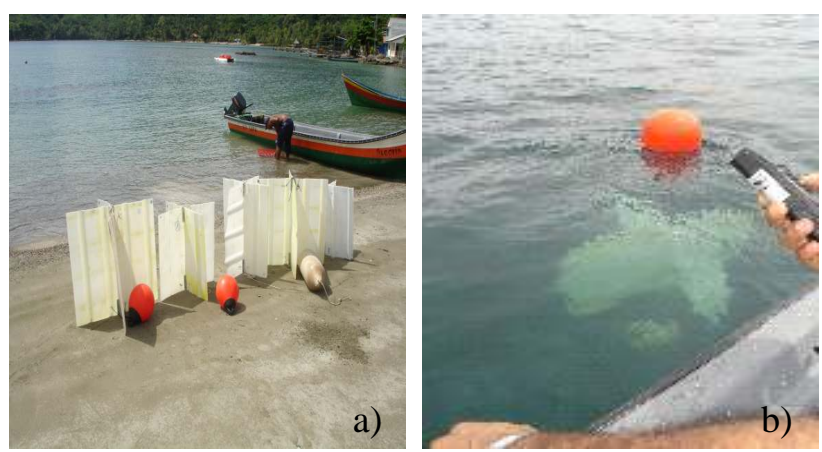

Figura 3. Boyas de deriva usadas para registrar información de campo. a) Estructura física. b) Monitoreo de cuerpos.

\section{Aplicación del modelo hidrodinámico RMA a la zona de estudio}

El modelo RMA es un modelo flexible que permite simular flujo y calidad de agua para ríos, bahías y sistemas estuarinos con influencia de marea, mediante la solución de las ecuaciones de Navier Stokes y conservación de masa en el régimen turbulento de Reynolds. La fricción con el fondo es calculada con la ecuación de Manning o Chezy [22] y permite elegir diferentes formulaciones para calcular los coeficientes de turbulencia. El esquema numérico de solución se basa en el método de elementos finitos, usa el método de los pesos residuales de Bubnov-Galerkin para representar las ecuaciones diferenciales. La integración espacial se realiza por el método de la cuadratura de Gauss. La solución numérica es completamente implícita y el conjunto de ecuaciones simultáneas resultantes es solucionado mediante el esquema no lineal de iteraciones de Newton-Raphson. El modelo está escrito en FORTRAN y puede ser usado en un computador personal $[23,24]$.
En la comprensión general del comportamiento del modelo y su respuesta a cambios en los parámetros físicos y numéricos, se realiza un análisis de sensibilidad por medio del método de perturbación de parámetros $[25,26]$ el cual consiste en representar la variable de observación, en términos de los parámetros físicos pertinentes, esto es $\mathrm{v}=f\left(x_{1}+x_{2}+\ldots \ldots x_{n}\right)$, donde $x_{i}$ es el conjunto de parámetros. En general, la sensibilidad de esta variable se observa en la respuesta del modelo bajo variaciones $\Delta x_{i}$ de uno de los parámetros físicos, respecto a valores típicos para sistemas marinos, dejando los otros parámetros constantes; el error en la predicción se calcula como:

$$
\Delta v=\frac{v\left(x_{i}+\Delta x_{i}\right)-v\left(x_{i}-\Delta x_{i}\right)}{2}
$$

Como se propone en Palacio y Toro [25] y atendiendo a las anotaciones en Wilkens [27] se evalúa la sensibilidad del modelo a parámetros numéricos tales como: dominio del modelo, escala temporal y escala espacial.

La calibración del modelo se realiza con el conjunto de datos correspondientes a la primera campaña de campo, con el objetivo de determinar los parámetros tanto físicos como numéricos [26] se inicia con una valoración subjetiva de los parámetros coeficiente de fricción de Manning, tamaño del dominio y de la malla, coeficiente de viscosidad turbulenta, paso de tiempo y condiciones de frontera; luego se seleccionan algunos parámetros relevantes, para ser optimizados mediante valoración objetiva la cual proporciona una medida cuantitativa del ajuste $S_{r}$, conocida como medida de error:

$$
S r=\sum_{i=1}^{n}\left(C_{p, i}-C_{m, i}\right)^{2}
$$

Donde $C_{p, i}$ y $C_{m, i}$ son el i-ésimo valor de la variable obtenidas del modelo y medida en campo respectivamente, $\mathrm{y} \mathrm{n}$ es el número de datos disponibles. Los parámetros son ajustados al menor valor de $S_{r}$ obtenido para el campo de velocidades del sistema.

Dado que los perfiles de velocidad, tomados en campo son de naturaleza lagrangiana y los resultados que presenta el modelo son eulerianos [28] se definen entonces intervalos espacio-temporales de 
observación de acuerdo con la información de campo disponible. Los resultados campo-modelo se comparan mediante el error cuadrático medio (rms):

$$
r m s=\sqrt{\sum_{i=1}^{n} \frac{\left(C_{p, i}-C_{m, i}\right)^{2}}{n}}
$$

Una vez calibrado el modelo, la validación se realiza comparando los resultados generados con los datos de campo correspondientes a la segunda campaña. Además se realiza una simulación de largo período que incluya varios ciclos de marea viva y muerta, para estudiar la respuesta de la velocidad en las bahías, respecto a la elevación del nivel del mar.

\section{Corrida del modelo en diversos escenarios}

Una vez se ha calibrado y verificado el modelo, se utiliza para predecir el comportamiento hidrodinámico de las bahías Sapzurro y Capurganá en diversas condiciones oceanográficas y climatológicas. Los resultados obtenidos son graficados y analizados con orientación hacia su aplicación en el campo de la protección ambiental y el aprovechamiento de la región.

\section{Resultados}

El régimen hidrodinámico modelado, se determinó de acuerdo a las características del sistema real y el tipo de información disponible para calibrar y verificar el modelo, obteniéndose la siguiente configuración física: flujo incompresible, horizontal y turbulento, aproximación de Boussinesq, batimetría constante, sin afluentes importantes para ser incluídos, no se consideran efectos de Coriolis y se adopta la formulación de Manning para la rugosidad del fondo. A continuación se presentan los resultados obtenidos de acuerdo con el modelo hidrodinámico implementado.

\section{Sensibilidad de los parámetros físicos}

Mediante la ejecución del modelo en estado estacionario se determinó la sensibilidad del campo de velocidad a variaciones del coeficiente de fricción de Manning y elevación del nivel del mar alrededor del valor típico correspondiente a las características de la zona de 0.025 y $0.25 \mathrm{~m}$ respectivamente. Los resultados son consignados en la tabla 1 , donde se presenta el promedio de los errores calculados para nodos de observación distribuidos sobre todo el dominio.
Tabla 1. Análisis de sensibilidad para el campo de velocidad respecto a los parámetros coeficiente de fricción de Manning ( 0.025$)$ y elevación $(0.25 \mathrm{~m})$. Errores promedio del campo de velocidades para variaciones de $5 \%, 10 \%$, $15 \%, 25 \%$ y $50 \%$ en los parámetros respecto a sus valores típicos.

\begin{tabular}{|llllll|}
\hline & $\begin{array}{l}\text { Promedio } \\
\text { error 5\% }\end{array}$ & $\begin{array}{l}\text { Promedio } \\
\text { error 10\% }\end{array}$ & $\begin{array}{l}\text { Promedio } \\
\text { error 15\% }\end{array}$ & $\begin{array}{l}\text { Promedio } \\
\text { error 25\% }\end{array}$ & $\begin{array}{l}\text { Promedio } \\
\text { error 50\% }\end{array}$ \\
\hline $\begin{array}{l}\text { Magnitud de la velocidad } \\
\text { respecto a coeficiente de fricción } \\
\text { de Manning. (mm/s) }\end{array}$ & 0.750 & 0.670 & 0.500 & 1.670 & 0.750 \\
$\begin{array}{l}\text { Dirección de la velocidad } \\
\text { respecto a coeficiente de fricción } \\
\text { de Manning. }\left(^{\circ}\right)\end{array}$ & 12.049 & 21.767 & 32.226 & 35.684 & 28.550 \\
$\begin{array}{l}\text { Magnitud de la velocidad } \\
\text { respecto a la elevación. }(\mathrm{mm} / \mathrm{s})\end{array}$ & 0.920 & 0.920 & 0.830 & 1.580 & 1.000 \\
$\begin{array}{l}\text { Dirección de la velocidad } \\
\text { respecto a la elevación }\left({ }^{\circ}\right)\end{array}$ & 16.803 & 19.354 & 25.661 & 57.132 & 27.556 \\
\hline
\end{tabular}

\section{Sensibilidad de los parámetros numéricos}

Primero se determinó la región del modelo que permite representar los fenómenos de interés en las bahías, partiendo de modelos individuales para cada una de ellas, luego se integraron en una única zona y por último se determinaron fronteras abiertas a una distancia promedio de $7 \mathrm{~km}$ mar adentro. Después de realizar varias simulaciones con diferentes pasos de tiempo asociados a números de Courant menores que uno, resultó posible usar pasos de tiempo del orden de horas. El análisis de sensibilidad para el tamaño de la malla, se focalizó en la zona de las bahías, indicando un tamaño de celda de lado $1 \mathrm{~m}$.

\section{Calibración}

Después de hacer una valoración subjetiva y objetiva, atendiendo el análisis de sensibilidad, para el coeficiente de Manning, se seleccionaron los tres valores de mejor ajuste para la magnitud de la velocidad que conservaron la tendencia en la dirección del patrón de flujo. Estos corresponden a $0.020,0.025$ y 0.030 . El resultado del residual promedio asociado a los nodos de observación (distribuidos sobre todo el dominio) es presentado en la tabla 2.

Tabla 2. Resultados de la calibración para la magnitud de la velocidad.

\begin{tabular}{|llll|}
\hline & $\begin{array}{l}\text { Coeficiente de } \\
\text { Manning }=\mathbf{0 . 0 2 0}\end{array}$ & $\begin{array}{l}\text { Coeficiente de } \\
\text { Manning =0.025 }\end{array}$ & $\begin{array}{l}\text { Coeficiente de } \\
\text { Manning = 0.030 }\end{array}$ \\
\hline Medida de Error $(\mathbf{m m} / \mathbf{s})$ & 0.92459 & 0.90725 & 0.83634 \\
\hline Valor $\mathbf{r m s}(\mathbf{m m} / \mathbf{s})$ & 6.08000 & 6.02000 & 5.78000 \\
\hline
\end{tabular}


Por último, se determinaron los parámetros físicos y numéricos de mejor ajuste para la magnitud de la velocidad que conservan la tendencia en la dirección del patrón de flujo consignados en la tabla 3. A pesar que el valor del coeficiente de fricción de Manning de 0.030 presenta el menor error respecto a la magnitud de los datos de campo, se conserva para el modelo el valor de 0.025 , esta elección se realizó conforme a mantener el menor error también en la dirección en zonas críticas del dominio. El modelo es ejecutado bajo el esquema de clausura de Smagorinsky para el cálculo de los términos de turbulencia.

Tabla 3. Parámetros del modelo.

\begin{tabular}{|ll|}
\hline Parámetro & Valor \\
\hline Resolución de la grilla & $\sim 100 \mathrm{~m}^{2}$ \\
Coeficiente de fricción de & 0.025 \\
Manning & \\
Aceleración de la gravedad & $9.8 \mathrm{~m} / \mathrm{s}$ \\
Paso de tiempo & 1 hora \\
Elevación promedio de marea & $0.25 \mathrm{~m}$ \\
\hline
\end{tabular}

\section{Verificación global del modelo}

Se corrió el modelo en estado dinámico con los parámetros establecidos en la época correspondiente a la segunda salida de campo (diciembre 19-22 de 2006), obteniéndose el patrón general del campo de velocidades. En las figuras 4-6 se presentan los resultados del modelo y los perfiles tomados en campo. Los diferentes cuerpos de deriva se identifican con colores distintos y la velocidad media lagrangiana se presenta en un recuadro y están dadas en $\mathrm{cm} / \mathrm{s}$.

Para examinar el comportamiento general del modelo, respecto a la marea se realiza una simulación de largo período cubriendo así varios períodos de marea viva marea muerta marea viva. Para el ciclo mostrado en la figura 7 , es evidente que la velocidad tanto en dirección como en magnitud en el orden obtenido del modelo, está fuertemente relacionada con la elevación de marea como se observa para la bahía de Sapzurro para el intervalo de llenado (ascenso de la onda de marea) y de vaciado (descenso de la onda de marea) en la figura 8. Este comportamiento se repite en simulaciones de corto período.

\section{Simulación de escenarios}

La simulación incluyendo efectos de viento y tormentas, genera cambios importantes en el patrón de circulación, por tanto, para usar el modelo con fines de pronóstico, es necesario entonces tener una buena descripción de las condiciones meteorológicas del lugar para lograr resultados confiables.
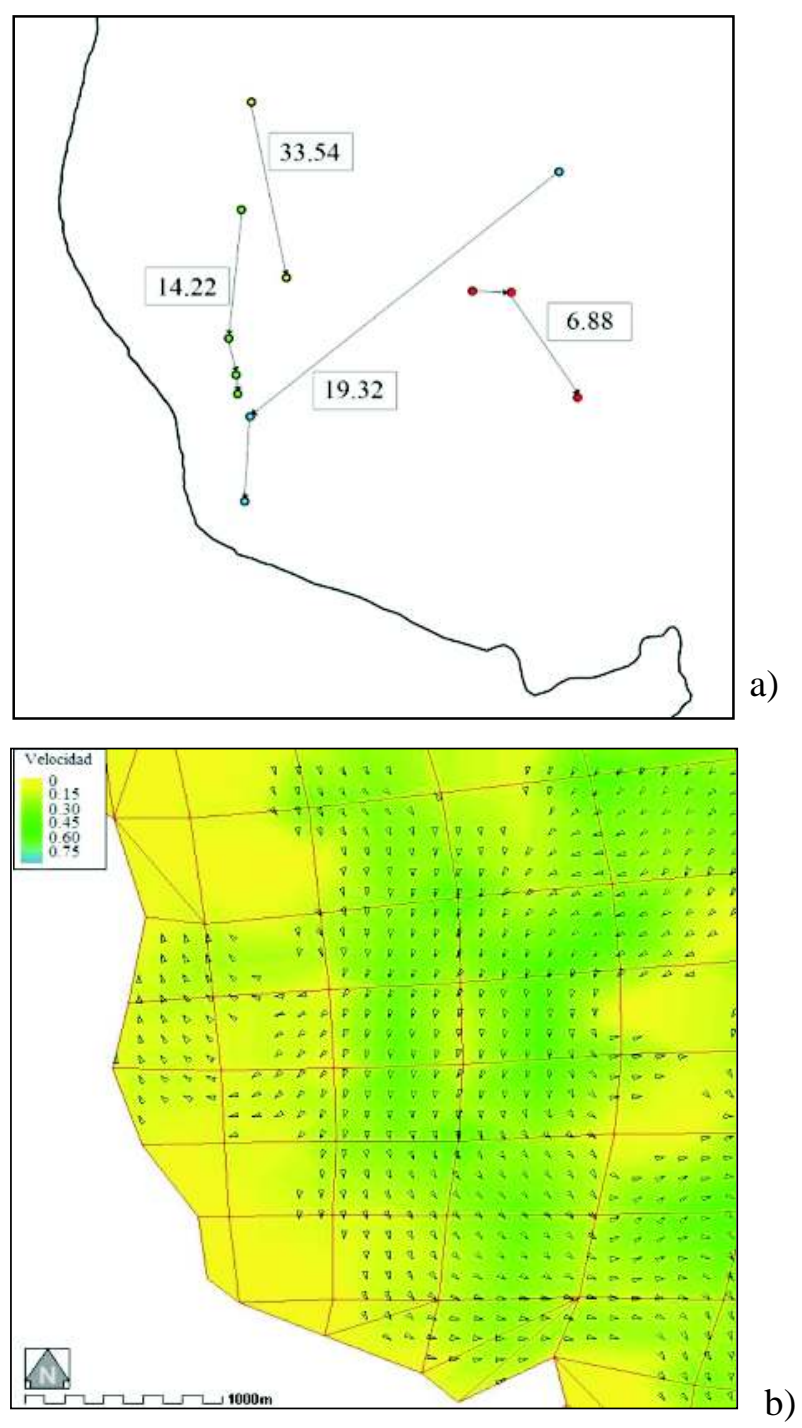

Figura 4. Perfiles de velocidad para el día 20/12/06 en el intervalo temporal 12-14 horas en la bahía de Capurganá. a) Mediciones de campo. El recorrido de cada boya se presenta con un color diferente y su velocidad media lagrangiana en un recuadro, las unidades son $\mathrm{cm} / \mathrm{s}$. b) Resultados obtenidos del modelo, las flechas indican la dirección del flujo, unidades de velocidad en $\mathrm{cm} / \mathrm{s}$. 

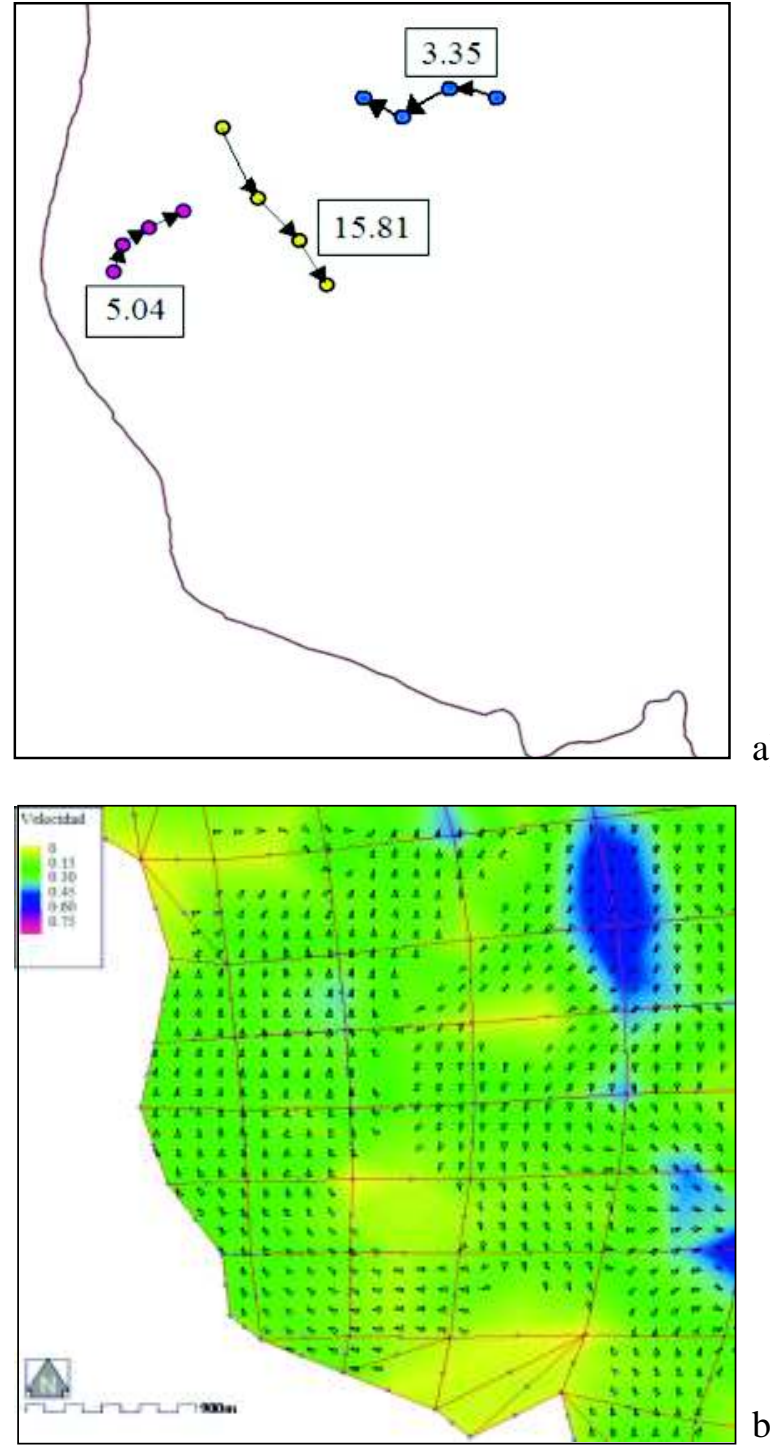

b)

Figura 5. Perfiles de velocidad para el día 20/12/06 en el intervalo temporal 14-16 horas en la bahía de Capurganá. a) Mediciones de campo. El recorrido de cada boya se presenta con un color diferente y su velocidad media lagrangiana en un recuadro, las unidades son $\mathrm{cm} / \mathrm{s}$. b) Resultados obtenidos del modelo, las flechas indican la dirección del flujo, unidades de velocidad en $\mathrm{cm} / \mathrm{s}$.

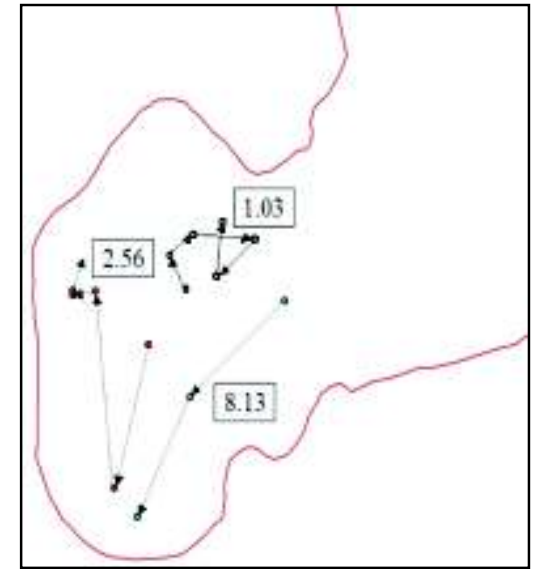

a)

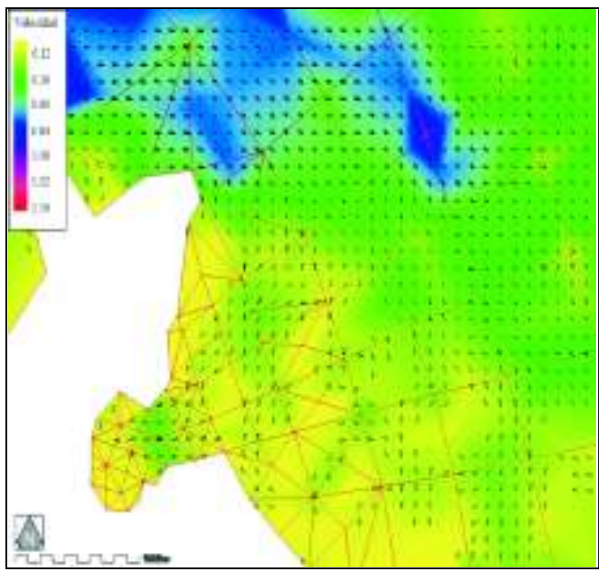

b)

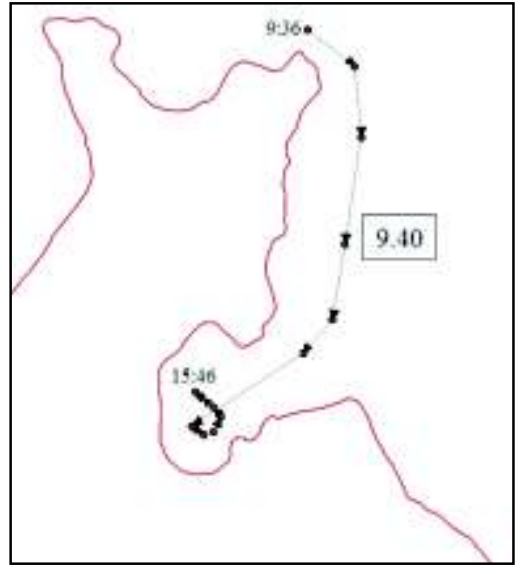

c)

Figura 6. Perfiles de velocidad para el día 22/12/06 en la bahía de Sapzurro. a) Mediciones de campo en el intervalo temporal 14-16 horas en la bahía de Sapzurro. El recorrido de cada boya se presenta con un color diferente y su velocidad media lagrangiana en un recuadro, las unidades son $\mathrm{cm} / \mathrm{s}$. b) Resultados obtenidos del modelo, las flechas indican la dirección del flujo, unidades de velocidad en $\mathrm{cm} / \mathrm{s}$. c) Tendencia general del movimiento del agua registrada con una boya desde las 9:36 hasta las 15:46 horas. 


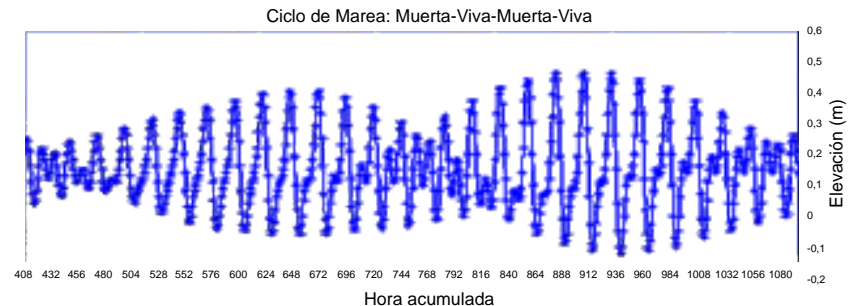

Figura 7. Ciclo marea viva-muerta de observación. Marea muerta 20/10/06, marea viva 28/10/06, marea muerta 01/11/06, marea viva 08/11/06.
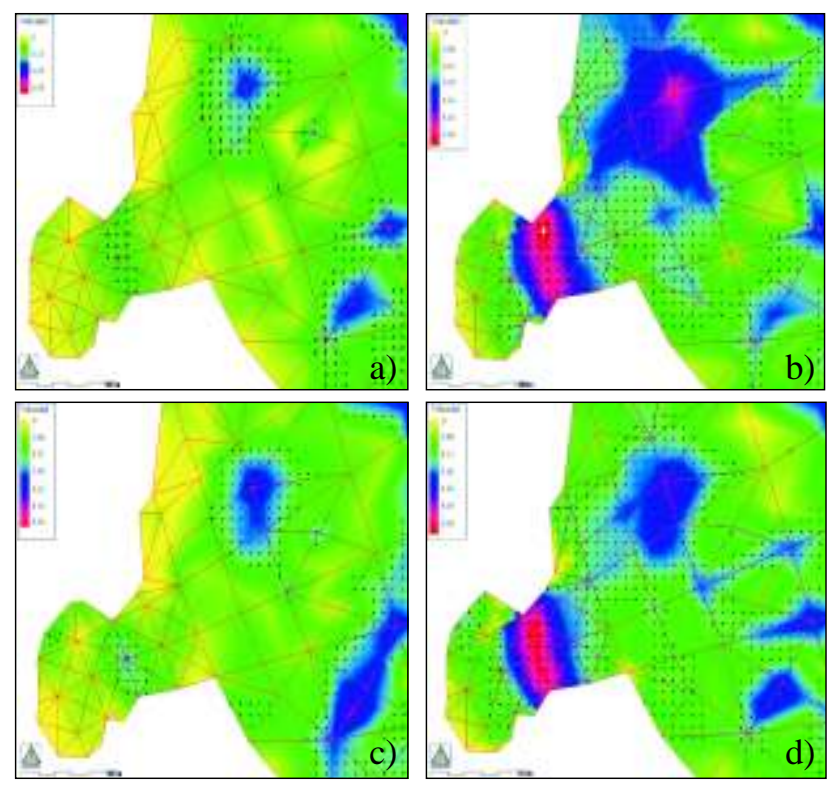

Figura 8. Comportamiento global de la bahía de Sapzurro en la fase de ascenso de marea, durante el ciclo: (a) Marea muerta 20/10/06. (b) Marea viva 28/10/06. (c) Marea muerta 01/11/06. (d) Marea viva 08/11/06.

\section{Comportamiento hidrodinámico derivado del modelo}

Se nota una tendencia general N-S en el flujo, bordeando la costa, desde Cabo Tiburón hasta Sapzurro, en la bahía de Sapzurro, la tendencia de movimiento es fuertemente influenciada por el estado de marea. Cuando la marea está subiendo, hay una corriente hacia el interior de la bahía, y cuando por el contrario, la marea baja, la corriente sale. Tanto en marea alta como en marea baja, se observa una disminución en la velocidad del movimiento del agua. Cuando el flujo está entrando en la bahía, el agua toma dos caminos: hacia el Norte, haciendo un giro sobre la costa y luego dependiendo de la onda de marea, el flujo se devuelve del mismo modo o sigue girando hasta encontrar salida hacia la boca de la bahía; si en ese punto, ya la marea está saliendo, entonces sigue a mar abierto, de lo contrario se queda circulando hasta ser nuevamente empujada hacia adentro. El otro camino es hacia el Sur y también gira sobre la costa, es este caso las velocidades son más pequeñas y con mayor posibilidad de quedarse en círculos ya que la zona sur de la bahía está protegida por un saliente. En el tramo Capurganá-Sapzurro, cercano a la costa se presentan corrientes cambiantes en sentido N-S y S-N, dependiendo también del estado de la marea. Cuando la marea está creciendo, el flujo entra a la bahía de Capurganá en dirección N-E, al chocar con la Punta del Aguacate, las líneas de corriente se dividen, unas siguen por fuera de la bahía y otras entran siguiendo la costa, con un giro en dirección S-N que nuevamente, dependiendo del período de la marea, pueden alcanzar la bahía de Sapzurro generando una corriente de entrada por el extremo sur de la boca de la bahía. Cuando la marea está descendiendo, la dirección general del flujo es saliendo, paralela a la costa en dirección sur.

\section{Discusión}

\section{Influencia de marea}

Los resultados del modelo muestran una importante influencia de la marea sobre el patrón de flujo de la zona en ausencia de viento, reflejado en corrientes que entran y salen de las bahías según el estado de la marea, lo cual está enteramente de acuerdo con las observaciones de campo. Dadas las diferencias morfológicas entre las bahías, este fenómeno se evidencia con mayor intensidad en la bahía de Sapzurro. En la bahía de Capurganá las corrientes de marea sufren deformaciones debido a la geometría propia de la bahía como se describió en el comportamiento hidrodinámico derivado del modelo. Este comportamiento resulta natural para este tipo de cuerpos de agua, donde, por su pequeño dominio espacial, se espera que el patrón de circulación esté dominado por efectos locales. Dado que para regiones costeras, las ondas de marea se comportan como ondas superficiales o de aguas someras, interactúan con el fondo de la cuenca produciendo de este modo una corriente importante. 


\section{Efectos morfológicos y batimétricos}

Las características batimétricas y morfológicas son también determinantes en la tendencia de la circulación, un ejemplo claro de esto es lo que se observa en la boca de la bahía de Sapzurro, donde se aprecia un aumento de la magnitud de la velocidad respecto al interior y exterior de la bahía, debido a que en comparación con estas zonas, la boca tiene menor área. Las velocidades en la bahía de Capurganá son en general mayores que en la bahía de Sapzurro lo cual se atribuye a las diferencias morfológicas entre ellas. En las ecuaciones de movimiento, el término de gradientes de presión, tiene un peso importante, hecho que se evidencia en el modelo al realizar una simulación sin forzamiento de marea, permitiendo identificar zonas de altas y bajas velocidades. En general, la coincidencia de la dirección del flujo de los resultados del modelo y las observaciones muestran la importancia de la geometría de la zona en la dirección del campo de velocidades.

\section{Diferencias entre magnitud de velocidades en campo y modeladas}

El modelo implementado, exhibe un campo de velocidades en general de dos órdenes de magnitud menor que los datos obtenidos en campo, lo cual es debido a los siguientes factores:

- Las velocidades medidas en campo, corresponden a datos referidos a la superficie del mar, se debe anotar que el perfil de velocidad en la columna de agua es en general parabólico, la forma exacta depende de parámetros físicos importantes como densidad, temperatura, tipo de mezcla y del coeficiente de viscosidad turbulento entre otros, para la región de estudio no se conoce la forma exacta del perfil vertical de velocidades. Los datos de velocidad obtenidos del modelo son promediados en la vertical, por tanto es de esperarse que sean menores que los valores superficiales de velocidad obtenidos con las boyas que aunque son cualitativamente valiosos porque son una guía experimental de los procesos reales en la circulación del agua, no son datos que puedan compararse directamente de una manera cuantitativa.

- Contribuyentes importantes localmente al comportamiento hidrodinámico de las bahías como velocidad del viento, estratificación del agua y flujos globales, no fueron incluidos en el modelo.

- Los parámetros físicos y numéricos fueron calculados como constantes para toda el área de estudio, sin importar las diferencias geomorfológicas presentadas al interior de las bahías, además fueron calculados con un conjunto de datos correspondientes a una sola época climática.

\section{Influencia del viento y clasificación de Hayes}

Según la clasificación de Hayes, que busca comparar los efectos del viento y de marea para establecer el fenómeno dominante en el patrón de circulación [27] se puede estimar el dominio para las bahías mediante la altura promedio de las olas generadas por viento y las ondas de marea. Para la zona de estudio, el rango promedio de altura de marea es $0.25 \mathrm{~m}$ y la altura media de las olas generadas por viento es de $1 \mathrm{~m}$, dando como resultado que la zona está dominada por efectos ondulatorios generados por viento. No se puede olvidar que los valores aquí utilizados, son promedios anuales, y en general obedecen al comportamiento del mar Caribe, por lo tanto esta es una tendencia global. Para la zona específica, se presentan variaciones a lo largo del año, dependiendo de las condiciones climáticas, pero la altura promedio de la marea no supera los $0.5 \mathrm{~m}$. En época de viento, éste se convierte en un factor determinante en el movimiento. El modelo permite introducir forzamiento de viento, lo cual altera significativamente el perfil de circulación. Para la zona no se encontraron datos certeros de vientos en las fechas de recolección de datos de campo, y por lo tanto la no inclusión de vientos en el modelo se perfila como una causa importante de error.

\section{Identificación de zonas potenciales de erosión y transporte de sedimentos}

Del comportamiento hidrodinámico obtenido del modelo, se identificaron zonas potenciales de erosión y sedimentación; además de los sitios típicos de erosión como salientes pronunciados, correspondientes a la zona 1 en la figura 9a, el modelo permite detectar posibles zonas de erosión y transporte de sedimentos. Al interior de la bahía de Sapzurro, se pueden distinguir las zonas 2 y 3 , figura 9 a. La primera corresponde a la parte norte, donde podría presentarse transporte continuo de sedimentos hacia la entrada de la bahía. La segunda zona, parte sur, a pesar de presentar velocidades más bajas y estar protegida por un saliente, también mueve sedimentos hacia la 
entrada de la bahía pero en menor cantidad. Una vez los sedimentos han alcanzado la entrada de la bahía, continuarán nuevamente con su ciclo, pero si la marea está bajando, los sedimentos se van hacia el mar. En la región de Cabo Tiburón figura 9a, zona 5, se presenta una tendencia general $\mathrm{N}-\mathrm{E}$, arrastrando consigo sedimentos en la misma dirección, este comportamiento además permite identificar una zona de posible depósito, correspondiente a la zona 4 en la figura (9a). Así mismo, pueden distinguirse dos zonas en Capurganá. La parte norte presenta flujo variable en dirección N-S, S-N dependiendo de la orientación de la marea. Cuando la corriente está en dirección S-N y alcanza a entrar en Sapzurro, el transporte de sedimentos está en esa dirección. Para el caso en que la dirección es N-S, el transporte de sedimentos es interrumpido por el lazo circular de corriente que se forma al sur de la bahía, los sedimentos salen al mar abierto por el centro, figura 9b. Aunque no se hicieron análisis de corrientes de deriva litoral, que es la encargada del transporte de sedimentos en la costa y producida por el oleaje, esta descripción está de acuerdo con los resultados obtenidos en Velásquez [11].

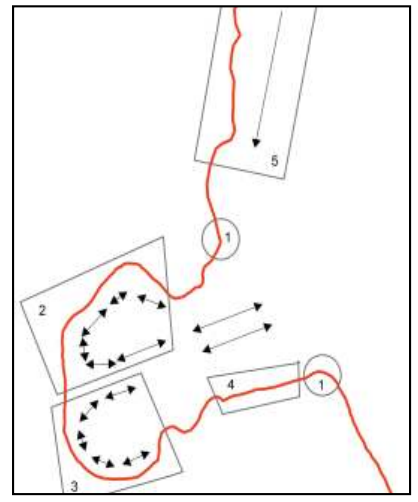

(a)

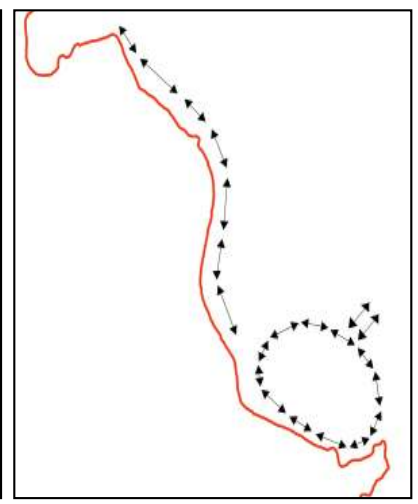

(b)
Figura 9. Esquema de identificación de zonas potenciales de erosión y transporte de sedimentos derivado de los resultados del modelo. (a) Sapzurro. (b) Capurganá.

\section{Agradecimientos}

El proyecto "Modelo hidrodinámico preliminar de las bahías Sapzurro y Capurganá, Darién Caribe colombiano" se realizó gracias al soporte económico del Comité para el Desarrollo de la Investigación CODI- de la Universidad de Antioquia, el Grupo de
Instrumentación Científica y Microelectrónica -GICM- del Instituto de Física y al aporte de material y equipos proporcionados por el Comité de Buceo Orcas, la Corporación Académica Ambiental -CAA- y El Grupo de Ingeniería y Gestión Ambiental-GIGA-.

\section{Referencias bibliográficas}

[1] Comisión Colombiana del Océano CCO. El océano en las ciencias naturales y sociales: espacio vital en la evolución de la humanidad y de Colombia. Bogotá (Colombia): Cassa Creativa Ltda; 2001.

[2] Comisión Colombiana del Océano CCO. Política nacional del océano y los espacios costeros. Santa Marta (Colombia): Litoflash; 2007.

[3] Bladé Castellet E. Modelación del flujo en lámina libre sobre cauces naturales: análisis integrado con esquemas en volúmenes finitos en una y dos dimensiones. Cataluña (España): Universidad Politécnica de Cataluña (Tesis doctoral); 2005.

[4] Stewart RH. Introduction to physical oceanography. Texas (USA): Texas A and M University; 2000.

[5] Lonin S, Anduckia JC, Parra C, Molares R. Sistema de pronóstico de las condiciones oceanográficas del mar Caribe. Boletín Científico CIOH 2003 Dic; (21):8-27.

[6] Lonin SA. Empleo de los datos de la NOAA, NODC, WOC en el modelo hidrodinámico del Caribe. Boletín Científico $\mathrm{CIOH}$ 2004 Dic; (22): 45-55.

[7] Molina A, Molina C, Chevillot P. La percepción remota aplicada para determinar la circulación de las aguas superficiales del golfo de Urabá y las variaciones de su línea de costa. Boletín Científico CIOH 1992 Jul; (11):43-58.

[8] Chevillot P, Molina A, Giraldo L, Molina C. Estudio geológico e hidrológico del golfo de Urabá. Boletín Científico CIOH 1993 Jul; (14):79-89.

[9] Montoya LJ, Toro FM, Bernal G. Dinámica oceanográfica del golfo de Urabá y su relación con la dispersión de sedimentos. En: Estudio de la dispersión de sedimento del río Atrato y sus impactos sobre la problemática ambiental costera del golfo de Urabá. Medellín (Colombia): Universidad Nacional de Colombia Escuela de Geociencias y Medio Ambiente (Informe Final); 2005. 11-27.

[10] Bernal G, Montoya F, Garizábal C, Toro M. La complejidad de la dimensión física en la problemática costera del Golfo de Urabá, Colombia. Gestión y Ambiente 2005 jul;8(1):123-135.

[11] Velásquez N. Geomorfología y dinámica de la línea de costa en el Urabá chocoano. Medellín (Colombia): Universidad Nacional de Colombia (Tesis); 2000. 
[12] Instituto de Hidrología, Meteorología y Estudios Ambientales IDEAM. y bajamares Costa Atlántica. Bogotá (Colombia): IDEAM; 2001.

[13] Grupo de Oceanografía Física de la Universidad de Vigo GOFUVI. Métodos en oceanografía física. Vigo (España): Universidad de Vigo; 2004.

[14] Correa T. Algunos aspectos de la dinámica poblacional de la cigua cittarium pica (Linnaeus, 1758), en el municipio de Acandí, Darién Caribe colombiano. Turbo (Colombia): Universidad de Antioquia (Tesis); 2006

[15] Valderrama D, Zea S. Esquema de distribución de esponjas arrecifales (porífera) del noroccidente del golfo de Urabá, Caribe Sur, Colombia. Boletín de Investigaciones Marinas y Costeras 2003;32:37-5.

[16] Pujos M, Pagliardini JL, Steer R, Vernette G, Weber O. Influencia de la contracorriente norte colombiana para la circulación de las aguas en la plataforma continental. Su acción sobre la dispersión de los efluentes en la suspensión del río Magdalena. Boletín Científico CIOH 1986 ene;(6):3-16.

[17] Andrade CA, Barton ED. Eddy development and motion in the Caribbean Sea. Journal of Geophysical Research 2000;105(C11):26191-26202.

[18] U.S. Army Corps of Engineers. Engineering and design coastal littoral transport. Washington (USA): Department of Army U. S. government Printing Office; 1992.

[19] Watson DF, Philip GM. A refinement of inverse distance weighted interpolation. Geoprocesing 1985;24:315-327.

[20] Vachon W. Current measurement by lagrangian drifting buoys: problems and potential. Oceans 1977;9:639-645.

[21] Davis R. An inexpensive drifter for surface currents. Current Measurement, Proceedings of the 1982 IEEE Second Working Conference on 1982;2:89-93.

[22] Rodríguez HA. Hidráulica experimental. 2da ed. Bogotá (Colombia): Escuela Colombiana de Ingeniería; 2002.

[23] King IP. Update documentation Rma2: A two dimensional finite element model for flow in estuaries and streams, version 7.4 A. Sydney (Australia): Resource Modelling Associates; 2004.

[24] King IP. Lectures about RMA model. Sydney (Australia): Resource Modelling Associates; 2004.

[25] Palacio CA, Toro M. Metodología para la validación de un modelo hidrodinámico específico en áreas intermareales. Avances en Recursos Hidráulicos 2002; 9:37-46.

[26] Chapra SC. Surface Water Quality Modelling. New York (USA): McGraw-Hill; 1997.

[27] Wilkens J. Medium scale morphodynamics of the Central Dithmarschen Bight. Kiel (Alemania): University of Kiel (Tesis doctoral); 2004.
[28] Gratton J. Introducción a la mecánica de fluidos. Buenos Aires (Argentina): CONICET-UBA; 2003. 